\title{
Peer support to encourage adoption of a Mediterranean diet: development of pilot randomised controlled intervention study protocol
}

\author{
C. T. McEvoy ${ }^{1}$, S. E. Moore ${ }^{1}$, L. Prior ${ }^{1}$, J. A. Lawton ${ }^{2}$, C. C. Patterson ${ }^{1}$, F. Kee ${ }^{1}$, M. Cupples ${ }^{1}$, \\ I. S. Young ${ }^{1}$, S. J. Hunter ${ }^{3}$, D. McCance ${ }^{3}$, K. Appleton ${ }^{4}$, M. C. McKinley ${ }^{1}$ and J. V. Woodside ${ }^{i}$ \\ ${ }^{1}$ Centre for Public Health, Queen's University Belfast, Belfast, BT12 6BJ, UK, ${ }^{2}$ Public Health Sciences, University of \\ Edinburgh Medical School, Edinburgh, EH8 9AG, UK, ${ }^{3}$ Regional Centre for Endocrinology and Diabetes, Belfast \\ Health and Social Care Trust, Belfast, BT12 6BA, UK and ${ }^{4}$ Psychology Research Centre, Bournemouth University, \\ Bournemouth, BH12 5BB, UK
}

A large randomised controlled trial demonstrated that adherence to a Mediterranean Diet (MD) significantly reduced risk of developing cardiovascular disease (CVD) ${ }^{(1)}$ using an intensive and expensive dietetic strategy to achieve dietary behaviour change. Peer support offers a potential low-cost approach $^{(2)}$ to encourage dietary behaviour change. However, there is considerable variation in how peer support (e.g. via group, mentor, telephone and/or web support) is delivered within intervention studies ${ }^{(3)}$.For a peer support programme to be effective it needs to be culturally sensitive and tailored to the target population ${ }^{(3)}$. This research aimed to explore the feasibility of peer support to encourage MD adoption in people at high CVD risk in Northern Ireland.

Twelve focus groups, homogenous with regards to gender, were conducted with the target population. Quantitative data was also recorded to determine individual preferred peer support approach. Group discussions were audio-recorded, transcribed verbatim and a thematic approach was used to analyse the data.

Sixty-seven high CVD risk adults (40 females, 27 males) took part in discussions. There was a high degree of acceptability for peer support to encourage MD change, with the majority $(89 \%)$ preferring a group-based approach. Women preferred group-based support in combination with telephone/mentor support, relative to men $(P=0.02)$.

\begin{tabular}{|c|c|c|c|c|c|c|c|}
\hline \multirow[b]{2}{*}{ Peer support approach* } & \multicolumn{2}{|l|}{ Total $(n=65)$} & \multicolumn{2}{|c|}{ Male $(n=25)$} & \multicolumn{2}{|c|}{ Female $(n=40)$} & \multirow[b]{2}{*}{$p$-value } \\
\hline & Mean Score & SD & Mean score & SD & Mean score & SD & \\
\hline Group & 1.5 & 1.04 & 1.6 & 1.12 & 1.5 & 1.01 & 0.61 \\
\hline Combination & 2.8 & 1.41 & 3.5 & 1.48 & 2.4 & 1.20 & 0.02 \\
\hline Peer mentor & 3.4 & 1.32 & 3.5 & 1.26 & 3.4 & 1.38 & 0.64 \\
\hline Telephone & 4.0 & 1.17 & 4.3 & 1.14 & 3.8 & 1.17 & 0.06 \\
\hline Web & 4.0 & 1.44 & 3.4 & 1.66 & 4.3 & 1.19 & 0.20 \\
\hline
\end{tabular}

* Ranked score where $1=$ most preferred peer support approach to $5=$ least preferred peer support approach.

It was felt that a group support approach should focus on discussion of shared experiences of changing toward a MD. Group sessions should also include practical, interactive MD education (e.g. tasting sessions, recipe ideas) delivered by peer supporters. In addition, several peer supporter characteristics and skills were described as being important to support people to change towards a MD. These included dietary knowledge, success in making dietary changes and possession of communication, interpersonal and group facilitation skills.

This research indicated preference for a group-based peer support approach to facilitate dietary change towards MD. A number of important factors were described, including preferred characteristics and skills for peer supporters and suggestions for the delivery of a group-based approach. These factors will be considered in the next stage of protocol development for a planned pilot randomised controlled intervention study.

1. Estruch R, Ros E, Salas-Salvadó J et al. (2013) NEngl JMed 368, 1279-1290.

2. Funnell MM (2010) Fam Pract 27, 17-21.

3. Gibson S (2007) Pub Health Nutr 10, 980-988. 